\title{
SIMPLE PERIODIC ORBITS OF MAPPINGS OF THE INTERVAL
}

BY

LOUIS BLOCK ${ }^{1}$

\begin{abstract}
Let $f$ be a continuous map of a closed, bounded interval into itself. A criterion is given to determine whether or not $f$ has a periodic point whose period is not a power of 2 , which just depends on the periodic orbits of $f$ whose period is a power of 2 . Also, a lower bound for the topological entropy of $f$ is obtained.
\end{abstract}

1. Introduction. Let $I$ denote a closed and bounded interval on the real line and let $C^{0}(I, I)$ denote the space of continuous maps from $I$ into itself. This paper is concerned with periodic orbits of mappings $f \in C^{0}(I, I)$. Such mappings (sometimes called first order difference equations) arise as mathematical models for phenomena in the natural sciences (see [4] and [5] for some discussion and further references).

Let $f \in C^{0}(I, I)$. Consider the following ordering of the positive integers:

$$
\begin{array}{r}
1,2,4,8, \ldots \quad \ldots, 7 \cdot 8,5 \cdot 8,3 \cdot 8, \ldots, 7 \cdot 4,5 \cdot 4,3 \cdot 4, \ldots, \\
7 \cdot 2,5 \cdot 2,3 \cdot 2, \ldots, 7,5,3 .
\end{array}
$$

A. N. Sarkovskii has proven that if $m$ is to the left of $n$ (in the above ordering) and $f$ has a periodic point of period $n$, then $f$ has a periodic point of period $m$ (see [6] or [7]). This theorem suggests that the following property implies a rich orbit structure:

(1) $f$ has a periodic point whose period is not a power of 2 .

This suggestion is supported by the fact that (1) implies the following:

(2) $f$ has a homoclinic point (see [1]);

(3) $f$ has positive topological entropy (see [1], [2], or [7]).

Also, (2) is equivalent to (1) (see [1]) and it has been conjectured (and proved for a special case in [3]) that (3) is equivalent to (1).

In this paper we give a criterion for determining whether or not $f$ satisfies (1) which just depends on the periodic orbits of $f$ whose period is a power of 2. In the process we obtain a lower bound for topological entropy. The criterion we give is based on the following definition.

Definition. Let $P$ be a periodic orbit of $f \in C^{0}(I, I)$ of period $m$, where $m$

Presented to the Society, November 12, 1978; received by the editors September 22, 1978.

AMS (MOS) subject classifications (1970). Primary 54H20.

${ }^{1}$ Partially supported by NSF Grant MCS 76-05822. 
is a power of 2 and $m \geqslant 2$. We say $P$ is simple if for any subset $\left\{q_{1}, \ldots, q_{n}\right\}$ of $P$ where $n$ divides $m$ and $n \geqslant 2$, and any positive integer $r$ which divides $m$, such that $\left\{q_{1}, \ldots, q_{n}\right\}$ is periodic orbit of $f^{r}$ with $q_{1}<q_{2}<\cdots<q_{n}$, we have

$$
f^{r}\left(\left\{q_{1}, \ldots, q_{n / 2}\right\}\right)=\left\{q_{n / 2+1}, \ldots, q_{n}\right\} .
$$

The reader may wish to see $\S 4$ where the definition of "simple" is discussed for a periodic orbit of period 8 , and some examples are given.

Our main results are the following: (In this paper we include $1=2^{0}$ as a power of 2.)

TheOREM A. Let $f \in C^{0}(I, I)$. $f$ has a periodic point whose period is not a power of 2 if and only if $f$ has a periodic orbit of period a power of 2 which is not simple.

TheOREM B. Let $f \in C^{0}(I, I)$. Suppose $f$ has a periodic orbit $P$ of period $\boldsymbol{m}$ (where $m=2^{k}$ for some $k \geqslant 2$ ) which is not simple. Then $f$ has a periodic point of period $3 \cdot 2^{k-2}$.

The proof of Theorems A and B uses some results of [6] and [7] which will be stated in §2. Stefan in [7] also obtains the following result which improves a theorem of Bowen and Franks (see [2]).

TheOREM C. Let $f \in C^{0}(I, I)$ and suppose $f$ has a periodic point of period $n$, where $n=2^{d} \cdot m$ and $m \geqslant 3$ is odd. Then the topological entropy of $f$ is greater than $\left(1 / 2^{d}\right) \log \sqrt{2}$.

Thus (using Theorem C) the following is an immediate corollary of Theorem B.

Corollary D. Let $f \in C^{0}(I, I)$. Suppose $f$ has a periodic orbit of period $m$ (where $m=2^{k}$ for some $k \geqslant 2$ ) which is not simple. Then the topological entropy of $f$ is greater than $\left(1 / 2^{k-2}\right) \log \sqrt{2}$.

2. Preliminary definitions and results. Let $f \in C^{0}(I, I)$ and let $N$ denote the set of positive integers. For any $n \in N$, we define $f^{n}$ inductively by $f^{1}=f$ and $f^{n}=f \circ f^{n-1}$. Let $f^{0}$ denote the identity map of $I$.

Let $x \in I$. $x$ is said to be a periodic point of $f$ if $f^{n}(x)=x$ for some $n \in N$. In this case the smallest element of $\left\{n \in N: f^{n}(x)=x\right\}$ is called the period of $x$.

We define the orbit of $x$ to be $\left\{f^{n}(x): n=0,1,2, \ldots\right\}$. If $x$ is a periodic point we say the orbit of $x$ is a periodic orbit, and we define the period of the orbit to be the period of $x$. Clearly, if $x$ is a periodic point of period $n$, then the orbit of $x$ contains $n$ points and each of these points is a periodic point of $f$ of period $n$. 
Note that a periodic point of $f$ is always a periodic point of $f^{n}$ (for any $n \in N$ ), but the periods may be different. The following proposition (which follows immediately from the definitions) gives an example of this.

Proposition 1. Let $f \in C^{0}(I, I)$. Suppose $P$ is a periodic orbit of $f$ of period $n$ where $n$ is even. Then there are disjoint subsets $P_{1}$ and $P_{2}$ of $P$ which are periodic orbits of $f^{2}$ of period $n / 2$.

Now, let $P$ be a periodic orbit of $f$ containing at least two points. Let $P_{\min }(f)$ denote the smallest element of $P$ and $P_{\max }(f)$ denote the largest element of $P$. Let

$$
U(f)=\{x \in I: f(x)>x\} \text { and } D(f)=\{x \in I: f(x)<x\} .
$$

Let $P_{U}(f)$ denote the largest element of $P \cap U(f)$ and $P_{D}(f)$ denote the smallest element of $P \cap D(f)$.

We will use the following lemma, proved by Štefan in [7] (see (9) in §B of [7]).

LeMma 2. Let $f \in C^{0}(I, I)$ and let $P$ be a periodic orbit of $f$. If $f$ has a fixed point between $P_{\min }(f)$ and $P_{U}(f)$ (or between $P_{D}(f)$ and $P_{\max }(f)$ ) then $f$ has periodic orbits of every period.

The following corollary to Lemma 2 also appears in [7].

LEMMa 3. Let $f \in C^{0}(I, I)$ and let $P$ be a periodic orbit of $f$. If $P_{D}(f)<$ $P_{U}(f)$ then $f$ has periodic orbits of every period.

Proof. Suppose $P_{D}(f)<P_{U}(f)$. Since $f\left(P_{D}(f)\right)<P_{D}(f)$ and $f\left(P_{U}(f)\right)>$ $P_{U}(f), f$ has a fixed point between $P_{D}(f)$ and $P_{U}(f)$. Thus, the hypothesis of Lemma 2 is satisfied. Q.E.D.

Lemma 4. Suppose $f \in C^{0}(I, I)$. Let $J \subset I$ and $K \subset I$ be closed intervals with $f(J) \supset K$. There is a closed interval $H \subset J$ with $f(H)=K$.

Proof. Let $K=[a, b]$ and let $A=f^{-1}(a) \cap J$ and $B=f^{-1}(b) \cap J$. Let $d$ denote the usual metric on the real line. Since $A$ and $B$ are nonempty disjoint compact sets, there are points $a_{1} \in A$ and $b_{1} \in B$ such that $d\left(a_{1}, b_{1}\right)=$ $d(A, B)$. Let $H$ be the closed interval with endpoints $a_{1}$ and $b_{1}$. Then $H \cap A=\left\{a_{1}\right\}$ and $H \cap B=\left\{b_{1}\right\}$. Hence $f(H)=K$. Q.E.D.

Lemma 5. Let $f \in C^{0}(I, I)$. Suppose $H$ and $K$ are closed intervals with $H \subset K \subset I$ and $f(H)=K$. Then $f$ has a fixed point in $H$.

Proof. Let $K=[a, b]$. For some $x \in H$ and $y \in H, f(x)=a$ and $f(y)=$ $b$. Hence $f(x) \leqslant x$ and $f(y) \geqslant y$. Thus, $f$ has a fixed point between $x$ and $y$. Q.E.D. 
LEMMA 6. Let $f \in C^{0}(I, I)$. Let $g=f^{r}$ for some positive integer $r$ which is a power of 2. Suppose there is a periodic orbit $P_{0}=\left\{q_{1}, \ldots, q_{n}\right\}$ of $g$ of period $n$, where $n$ is a power of 2 and $n \geqslant 2$. Suppose $q_{1}<q_{2}<\cdots<q_{n}$ and for some $i<n / 2$ and $j \leqslant n / 2, g\left(q_{i}\right)=q_{j}$. Then there is a periodic orbit $P$ of $f$ of period a power of 2 which is not simple.

Proof. Let $P$ be the orbit of $q_{1}$ with respect to $f$. Then $P$ is a periodic orbit and $P_{0} \subset P$. Let $m$ be the period of $P$. We will show that $m=n \cdot r$.

We claim that, for any positive integer $s<r$ and any $q_{j} \in P_{0}, f^{s}\left(q_{j}\right) \notin P_{0}$. To prove this, suppose that, for some positive integer $s<r$ and some $q_{j} \in P_{0}$, $f^{s}\left(q_{j}\right) \in P_{0}$. We may assume (by choosing $s$ smaller if necessary) that, for any positive integer $t<s, f^{t}\left(q_{i}\right) \notin P_{0}$ for $i=1, \ldots, n$. Note that for $k=$ $0, \ldots, n-1$,

$$
f^{s}\left(f^{k \cdot r}\left(q_{j}\right)\right)=f^{k \cdot r}\left(f^{s}\left(q_{j}\right)\right) \in P_{0}
$$

Hence $f^{s}\left(P_{0}\right) \subset P_{0}$. Since $f$ restricted to $P$ is one-to-one, $f^{s}\left(P_{0}\right)=P_{0}$. Since $f^{r}\left(P_{0}\right)=P_{0}$ and $s<r$, it follows from the choice of $s$ that $s$ divides $r$.

Now, $f^{s}\left(P_{0}\right)=P_{0}$ implies that some subset $P_{1}$ of $P_{0}$ is a periodic orbit of $f^{s}$. Hence $f^{s}\left(P_{1}\right)=P_{1}$. Since $s$ divides $r, f^{r}\left(P_{1}\right)=P_{1}$. Hence $P_{1}=P_{0}$. Thus $P_{0}$ is a periodic orbit of $f^{s}$ and a periodic orbit of $f^{r}$. Since $r$ is a power of $2, s$ divides $r, s<r$, and $P_{0}$ has at least two elements, we obtain a contradiction by repeated application of Proposition 1. This contradiction establishes our claim.

Next, we will show that the points

$$
q_{1}, \ldots, q_{n}, f\left(q_{1}\right), \ldots, f\left(q_{n}\right), \ldots, f^{r-1}\left(q_{1}\right), \ldots, f^{r-1}\left(q_{n}\right)
$$

are all distinct. Suppose $f^{a}\left(q_{i}\right)=f^{b}\left(q_{j}\right)$ where $0<a \leqslant r-1,0 \leqslant b \leqslant r-1$, $1 \leqslant i \leqslant n$, and $1 \leqslant j \leqslant n$. We may assume that $a \leqslant b$. By applying $f^{r-b}$ to the points $f^{a}\left(q_{i}\right)$ and $f^{b}\left(q_{j}\right)$, we see that $f^{r-b+a}\left(q_{i}\right) \in P_{0}$. Since $a<b, r-b+$ $a<r$. By our claim above, $r-b+a=r$. Hence $a=b$. Again, applying $f^{r-b}$ to the points $f^{a}\left(q_{i}\right)$ and $f^{b}\left(q_{j}\right)$ we see that $f^{r}\left(q_{i}\right)=f^{r}\left(q_{j}\right)$. Since $f^{r}$ restricted to $P_{0}$ is one-to-one, $q_{i}=q_{j}$. Hence $i=j$.

Clearly,

$$
P=\left\{q_{1}, \ldots, q_{n}, f\left(q_{1}\right), \ldots, f\left(q_{n}\right), \ldots, f^{r-1}\left(q_{1}\right), \ldots, f^{r-1}\left(q_{n}\right)\right\} .
$$

Hence $m=n \cdot r$. Thus $m$ is a power of 2 and $r$ divides $m$. It follows from this and our hypothesis that $P$ is not simple. Q.E.D.

\section{Proof of Theorems A and B.}

LEMMA 7. Let $f \in C^{0}(I, I)$. Let $n \geqslant 3$ be an odd integer and suppose that $f^{n}$ does not have any periodic orbits of period 3. Let $P$ be a periodic orbit of $f$ of period $k$, where $k$ is a power of 2 and $k \geqslant 2$. Then $P_{U}\left(f^{n}\right)=P_{U}(f)$ and $P_{D}\left(f^{n}\right)=P_{D}(f)$ 
Proof. Note that $P_{U}\left(f^{n}\right)$ and $P_{D}\left(f^{n}\right)$ are well defined because $P$ is a periodic orbit of $f^{n}$.

Our hypothesis implies that $f$ does not have any periodic orbits of period 3. $n$. By Lemma 3, $P_{U}(f)<P_{D}(f)$. It follows from this (and the definitions of $P_{U}(f)$ and $\left.P_{D}(f)\right)$ that there are no elements of $P$ between $P_{U}(f)$ and $\boldsymbol{P}_{D}(f)$. Also, by Lemma $3, \boldsymbol{P}_{U}\left(f^{n}\right)<\boldsymbol{P}_{D}\left(f^{n}\right)$ and there are no elements of $\boldsymbol{P}$ between $P_{U}\left(f^{n}\right)$ and $P_{D}\left(f^{n}\right)$.

It suffices to prove that $P_{U}\left(f^{n}\right)=P_{U}(f)$. Suppose $P_{U}\left(f^{n}\right) \neq P_{U}(f)$. We have two cases.

Case 1. $P_{U}(f)<P_{U}\left(f^{n}\right)$. Then $P_{U}(f)<P_{D}(f)<P_{U}\left(f^{n}\right)<P_{D}\left(f^{n}\right)$. Since $f\left(P_{\text {min }}(f)\right)>P_{\min }(f)$ and $f\left(P_{D}(f)\right)<P_{D}(f), f$ has a fixed point between $P_{\min }(f)$ and $P_{D}(f)$. Hence, $f^{n}$ has a fixed point between $P_{\min }\left(f^{n}\right)=P_{\min }(f)$ and $P_{U}\left(f^{n}\right)$. By Lemma $2, f^{n}$ has periodic orbits of every period, a contradiction.

Case 2. $P_{U}\left(f^{n}\right)<P_{U}(f)$. Then $P_{U}\left(f^{n}\right)<P_{D}\left(f^{n}\right)<P_{U}(f)<P_{D}(f)$. It follows that $f$ has a fixed point between $P_{U}(f)$ and $P_{\max }(f)$, so $f^{n}$ has a fixed point between $P_{D}\left(f^{n}\right)$ and $P_{\max }\left(f^{n}\right)$. By Lemma $2, f^{n}$ has periodic orbits of every period, a contradiction. Q.E.D.

Lemma 8. Let $f \in C^{0}(I, I)$. Let $P=\left\{p_{1}, \ldots, p_{n}\right\}$ be a periodic orbit of $f$ of period $n$, where $n$ is a power of 2 and $n \geqslant 2$, and $p_{1}<p_{2}<\cdots<p_{n}$. Suppose that, for every odd positive integer $m<n, f^{m}$ does not have any periodic orbits of period 3. Then

$$
f\left(\left\{p_{1}, \ldots, p_{n / 2}\right\}\right)=\left\{p_{n / 2+1}, \ldots, p_{n}\right\}
$$

and

$$
f\left(\left\{p_{n / 2+1}, \ldots, p_{n}\right\}\right)=\left\{p_{1}, \ldots, p_{n / 2}\right\}
$$

Proof. We claim that $f(P \cap U(f)) \subset P \cap D(f)$. Suppose the claim is false. Then for some $p_{0} \in P \cap U(f), f\left(p_{0}\right) \in P \cap U(f)$. Let $k$ be the smallest nonnegative integer with $f^{k}\left(f\left(p_{0}\right)\right)=p_{1}$. Note that $1<k<n$.

If $k$ is odd then $p_{1}<f\left(p_{0}\right)$ and $f^{k}$ has a fixed point between $p_{1}$ and $f\left(p_{0}\right)$. Now $f\left(p_{0}\right) \leqslant P_{U}(f)$ and, by Lemma $7, P_{U}(f)=P_{U}\left(f^{k}\right)$. Hence, $f^{k}$ has a fixed point between $P_{\min }\left(f^{k}\right)$ and $P_{U}\left(f^{k}\right)$. By Lemma $2, f^{k}$ has periodic orbits of every period. This contradicts our hypothesis.

If $k$ is even then $k+1$ is odd, $k+1<n$, and $f^{k+1}\left(p_{0}\right)=p_{1}$. Hence, $p_{1}<p_{0}$ and $f^{k+1}$ has a fixed point between $p_{1}$ and $p_{0}$. By Lemma $7, f^{k+1}$ has a fixed point between $P_{\min }\left(f^{k+1}\right)$ and $P_{U}\left(f^{k+1}\right)$. Again, using Lemma 2, we obtain a contradiction.

This establishes our claim that $f(P \cap U(f)) \subset P \cap D(f)$. By a similar proof, it follows that $f(P \cap D(f)) \subset P \cap U(f)$. Since the restriction of $f$ to $P$ is a bijection it follows that 


$$
f(P \cap U(f))=P \cap D(f) \text { and } f(P \cap D(f))=P \cap U(f) .
$$

Hence $P \cap U(f)$ and $P \cap D(f)$ have an equal number of points. Since $P_{U}(f)<P_{D}(f)$ by Lemma 3, this proves Lemma 8. Q.E.D.

Proposition 9. Let $f \in C^{0}(I, I)$. Suppose $\left\{p_{1}, \ldots, p_{n}\right\}$ is a periodic orbit of $f$ of period $n$, where $n$ is a power of 2 and $n \geqslant 2$. Suppose $p_{1}<p_{2}<\cdots<p_{n}$ and $f\left(\left\{p_{1}, \ldots, p_{n / 2}\right\}\right) \neq\left\{p_{n / 2+1}, \ldots, p_{n}\right\}$. Then $f$ has $a$ periodic point of period $s$, where $s$ is odd and $3 \leqslant s \leqslant 3(n-1)$.

Proof. By hypothesis and Lemma 8 , for some odd integer $m<n, f^{m}$ has a periodic point of period 3. The conclusion follows easily from this. Q.E.D.

Lemma 10. Let $f \in C^{0}(I, I)$. Suppose $f$ has a periodic orbit $\left\{p_{1}, p_{2}, p_{3}, p_{4}\right\}$ with $p_{1}<p_{2}<p_{3}<p_{4}$ and $f\left(\left\{p_{1}, p_{2}\right\}\right) \neq\left\{p_{3}, p_{4}\right\}$. Then $f$ has a periodic point of period 3 .

Proof. Let $I_{1}=\left[p_{1}, p_{2}\right], I_{2}=\left[p_{2}, p_{3}\right]$, and $I_{3}=\left[p_{3}, p_{4}\right]$. Our hypothesis implies that either $f\left(p_{1}\right)=p_{2}$ or $f\left(p_{2}\right)=p_{1}$.

Case 1. $f\left(p_{1}\right)=p_{2}$. Since $f\left(p_{2}\right)=p_{3}$ or $f\left(p_{2}\right)=p_{4}$, we have $f\left(I_{1}\right) \supset I_{2}$. Also, since $f\left(p_{2}\right)=p_{4}$ or $f\left(p_{3}\right)=p_{4}$. we have $f\left(I_{2}\right) \supset I_{3}$. Finally, since $f\left(p_{3}\right)=p_{1}$ or $f\left(p_{4}\right)=p_{1}$, we have $f\left(I_{3}\right) \supset I_{1}$.

By Lemma 4, there are closed intervals $J_{1} \subset I_{1}, J_{2} \subset I_{2}$, and $J_{3} \subset I_{3}$ such that $f\left(J_{3}\right)=I_{1}, f\left(J_{2}\right)=J_{3}$, and $f\left(J_{1}\right)=J_{2}$. It follows that $f^{3}\left(J_{1}\right)=I_{1}$. By Lemma $5, f^{3}$ has a fixed point $x \in J_{1}$. Since $f(x) \in I_{2}, x$ is a periodic point of $f$ of period 3 .

Case 2. $f\left(p_{2}\right)=p_{1}$. Then $f\left(p_{1}\right)=p_{3}$ or $f\left(p_{1}\right)=p_{4}$. Thus, $f\left(I_{1}\right) \supset I_{1}$, and $f\left(I_{1}\right) \supset I_{2}$. Also, $f\left(p_{2}\right)=p_{1}$ implies that $f\left(I_{2}\right) \supset I_{1}$. By Lemma 4, there are closed intervals $J_{1} \subset I_{1}, J_{2} \subset I_{1}$, and $J_{3} \subset I_{2}$ such that $f\left(J_{3}\right)=I_{1}, f\left(J_{2}\right)=J_{3}$, and $f\left(J_{1}\right)=J_{2}$. It follows that $f^{3}\left(J_{1}\right)=I_{1}$. By Lemma $5, f^{3}$ has a fixed point $x \in J_{1}$. Since $f^{2}(x) \in I_{2}, x$ is a periodic point of $f$ of period 3. Q.E.D.

TheOREM B. Let $f \in C^{0}(I, I)$. Suppose $f$ has a periodic orbit $P$ of period $m$ (where $m=2^{k}$ for some $k \geqslant 2$ ) which is not simple. Then $f$ has a periodic point of period $3 \cdot 2^{k-2}$.

Proof. By hypothesis there is a subset $\left\{q_{1}, \ldots, q_{n}\right\}$ of $P$ and a positive integer $r$ which divides $m$ such that $\left\{q_{1}, \ldots, q_{n}\right\}$ is a periodic orbit of $f^{r}$ with $q_{1}<q_{2}<\cdots<q_{n}$ and

$$
f^{r}\left(\left\{q_{1}, \ldots, q_{n / 2}\right\}\right) \neq\left\{q_{n / 2+1}, \ldots, q_{n}\right\} .
$$

This implies $n>2$. It follows from the proof of Lemma 6 that $m=n \cdot r$. Hence $r \leqslant 2^{k-2}$.

First suppose $r=2^{k-2}$. Then $n=4$, so $\left\{q_{1}, q_{2}, q_{3}, q_{4}\right\}$ is a periodic orbit of $f^{r}$ of period 4 with $f^{r}\left(\left\{q_{1}, q_{2}\right\}\right) \neq\left\{q_{3}, q_{4}\right\}$. By Lemma $10, f^{r}$ has a periodic 
point of period 3. By the theorem of Šarkovskii (stated in $\S 1$ ), $f$ has a periodic point of period $3 \cdot r=3 \cdot 2^{k-2}$.

Now suppose $r<2^{k-2}$. Then $r \leqslant 2^{k-3}$. By Proposition $9, f^{r}$ has a periodic point of period $s$, where $s$ is odd and $s>3$. By the theorem of Šarkovskii, $f$ has a periodic point of period $3 \cdot 2^{k-2}$. Q.E.D.

TheOREM A. Let $f \in C^{0}(I, I)$. $f$ has a periodic point whose period is not a power of 2 if and only if $f$ has periodic orbit of period a power of 2 which is not simple.

Proof. The "if" part of the theorem follows from Theorem B.

Suppose $f$ has a periodic point whose period is not a power of 2. By the theorem of Sarkovskií, stated in $\S 1$, for some positive integer $r$ which is a power of $2, f^{r}$ has a periodic orbit $P$ of period 3. Let $P=\left\{p_{1}, p_{2}, p_{3}\right\}$ with $p_{1}<p_{2}<p_{3}$.

Let $g=f^{r}$. Then $g\left(p_{1}\right)=p_{2}$ or $g\left(p_{3}\right)=p_{2}$. We may assume without loss of generality that $g\left(p_{1}\right)=p_{2}$. This implies that $g\left(p_{2}\right)=p_{3}$ and $g\left(p_{3}\right)=p_{1}$.

Since $g\left(p_{2}\right)>p_{2}$ and $g\left(p_{3}\right)<p_{3}, g$ has a fixed point $e \in\left(p_{2}, p_{3}\right)$. Let $I_{1}=\left[p_{1}, p_{2}\right], I_{2}=\left[p_{2}, e\right]$, and $I_{3}=\left[e, p_{3}\right]$. Then $g\left(I_{1}\right) \supset I_{2}, g\left(I_{1}\right) \supset I_{3}, g\left(I_{2}\right)$ $\supset I_{3}, g\left(I_{3}\right) \supset I_{1}$, and $g\left(I_{3}\right) \supset I_{2}$. By Lemma 4, there are closed intervals $J_{8} \subset I_{3}$ with $g\left(J_{8}\right)=I_{1}, J_{7} \subset I_{2}$ with $g\left(J_{7}\right)=J_{8}, J_{6} \subset I_{3}$ with $g\left(J_{6}\right)=J_{7}$, $J_{5} \subset I_{2}$ with $g\left(J_{5}\right)=J_{6}, J_{4} \subset I_{1}$ with $g\left(J_{4}\right)=J_{5}, J_{3} \subset I_{3}$ with $g\left(J_{3}\right)=J_{4}$, $J_{2} \subset I_{2}$ with $g\left(J_{2}\right)=J_{3}$, and $J_{1} \subset I_{1}$ with $g\left(J_{1}\right)=J_{2}$.

It follows that $g^{8}\left(J_{1}\right)=I_{1}$. By Lemma $5, g^{8}$ has a fixed point $c \in J_{1}$. Hence $c$ is a periodic point of $g$ of period $1,2,4$, or 8 . Since $g(c) \in I_{2}, g^{2}(c) \in I_{3}$, and $g^{4}(c) \in I_{2}, c$ is a periodic point of $g$ of period 8 .

Let $\left\{q_{1}, \ldots, q_{8}\right\}$ denote the orbit of $c$ where $q_{1}<q_{2}<\cdots<q_{8}$. We claim that, for some $i \leqslant 4$ and $j \leqslant 4, g\left(q_{i}\right)=q_{j}$. Note that $c \in I_{1}, g^{3}(c) \in I_{1}$, $g(c) \in I_{2}, g^{4}(c) \in I_{2}, g^{6}(c) \in I_{2}, g^{2}(c) \in I_{3}, g^{5}(c) \in I_{3}$, and $g^{7}(c) \in I_{3}$. Hence, $\left\{q_{1}, q_{2}, q_{3}, q_{4}\right\}$ contains $c, g^{3}(c)$, and two of the points $g(c), g^{4}(c)$, and $g^{6}(c)$.

First, suppose that $g(c) \in\left\{q_{1}, q_{2}, q_{3}, q_{4}\right\}$. Then the claim is true with $q_{i}=c$ and $q_{j}=g(c)$. Now, suppose that $g(c) \notin\left\{q_{1}, q_{2}, q_{3}, q_{4}\right\}$. Then $g^{4}(c) \in$ $\left\{q_{1}, q_{2}, q_{3}, q_{4}\right\}$. So the claim holds with $q_{i}=g^{3}(c)$ and $q_{j}=g^{4}(c)$.

Thus, our claim holds in either case. By Lemma $6, f$ has a periodic orbit of period a power of 2 which is not simple. Q.E.D.

4. Some examples. Let $f \in C^{0}(I, I)$ and let $P=\left\{p_{1}, \ldots, p_{8}\right\}$ be a periodic orbit of $f$ of period 8 with $p_{1}<p_{2}<\cdots<p_{8}$. Then $P$ is simple if and only if the following two conditions hold:

(1) $f\left(\left\{p_{1}, p_{2}, p_{3}, p_{4}\right\}\right)=\left\{p_{5}, p_{6}, p_{7}, p_{8}\right\}$,

(2) $f^{2}\left(\left\{p_{1}, p_{2}\right\}\right)=\left\{p_{3}, p_{4}\right\}$ and $f^{2}\left(\left\{p_{5}, p_{6}\right\}\right)=\left\{p_{7}, p_{8}\right\}$.

Clearly, (1) and (2) and the fact that $P$ is a periodic orbit of period 8 imply 
that

$$
\begin{gathered}
f\left(\left\{p_{5}, p_{6}, p_{7}, p_{8}\right\}\right)=\left\{p_{1}, p_{2}, p_{3}, p_{4}\right\}, \\
f^{2}\left(\left\{p_{3}, p_{4}\right\}\right)=\left\{p_{1}, p_{2}\right\}, \quad f^{2}\left(\left\{p_{7}, p_{8}\right\}\right)=\left\{p_{5}, p_{6}\right\}, \\
f^{4}\left(p_{1}\right)=p_{2}, \quad f^{4}\left(p_{2}\right)=p_{1}, \quad f^{4}\left(p_{3}\right)=p_{4}, \quad f^{4}\left(p_{4}\right)=p_{3}, \\
f^{4}\left(p_{5}\right)=p_{6}, \quad f^{4}\left(p_{6}\right)=p_{5}, \quad f^{4}\left(p_{7}\right)=p_{8}, \quad f^{4}\left(p_{8}\right)=p_{7} .
\end{gathered}
$$

EXAMPLE 1. $f\left(p_{1}\right)=p_{5}, f\left(p_{2}\right)=p_{6}, f\left(p_{3}\right)=p_{8}, f\left(p_{4}\right)=p_{7}, f\left(p_{5}\right)=p_{3}, f\left(p_{6}\right)$ $=p_{4}, f\left(p_{7}\right)=p_{1}$, and $f\left(p_{8}\right)=p_{2}$.

In this example $P$ is simple.

EXAMPLE 2. $f\left(p_{1}\right)=p_{2}, f\left(p_{2}\right)=p_{5}, f\left(p_{3}\right)=p_{7}, f\left(p_{4}\right)=p_{8}, f\left(p_{5}\right)=p_{3}, f\left(p_{6}\right)$ $=p_{1}, f\left(p_{7}\right)=p_{4}$, and $f\left(p_{8}\right)=p_{6}$.

In this example $P$ is not simple because condition (1) above does not hold. By Proposition 9, $f$ has a periodic point of period $s$, where $s$ is odd and $3<s<21$. By Theorem $\mathrm{C}$ of $\S 1$, the topological entropy of $f$ is greater than $\log \sqrt{2}$.

EXAMPLE 3. $f\left(p_{1}\right)=p_{5}, f\left(p_{2}\right)=p_{7}, f\left(p_{3}\right)=p_{6}, f\left(p_{4}\right)=p_{8}, f\left(p_{5}\right)=p_{2}, f\left(p_{6}\right)$ $=p_{4}, f\left(p_{7}\right)=p_{3}$, and $f\left(p_{8}\right)=p_{1}$.

In this example $P$ is not simple because condition (2) does not hold (since $\left.f^{2}\left(p_{1}\right)=p_{2}\right)$. By Theorem B, $f$ has a periodic point of period 6 , and by Corollary $\mathrm{D}$, the topological entropy of $f$ is greater than $\left(\frac{1}{2}\right) \log \sqrt{2}$.

\section{REFERENCES}

1. L. Block, Homoclinic points of mappings of the interval, Proc. Amer. Math. Soc. 72 (1978), 576-580.

2. R. Bowen and J. Franks, The periodic points of maps of the disc and the interval, Topology 15 (1976), 337-442.

3. L. Jonker and D. Rand, $A$ lower bound for the entropy of certain maps of the unit interval (preprint).

4. T. Li and J. A. Yorke, Period three implies chaos, Amer. Math. Monthly 82 (1975), 985-992.

5. R. M. May, Simple mathematical models with very complicated dynamics, Nature 261 (1976), 459-467.

6. A. N. Sarkovskii, Coexistence of cycles of a continuous map of a line into itself, Ukrain. Mat. Z. 16 (1964), 61-71.

7. P. Stefan, A theorem of Sarkouskii on the existence of periodic orbits of continuous endomorphisms of the real line, Comm. Math. Phys. 54 (1977), 237-248.

Department of Mathematics, University of Florida, Gainesville, Florma 32611 